\title{
Is mean platelet volume an inflammatory marker in acne patients treated with isotretinoin?
}

\author{
Funda Tamer ${ }^{1 凶}$, Mehmet Eren Yuksel ${ }^{2}$, Emine Avcl ${ }^{3}$
}

\begin{abstract}
Introduction: Isotretinoin is a commonly used systemic retinoid for treating acne. However, isotretinoin may lead to elevated serum levels of triglycerides and cholesterol, and it may affect liver function tests. Moreover, the effect of isotretinoin on hematological parameters remains controversial. This study examines changes in the blood chemistry panel, hematological parameters, and inflammation biomarkers of patients diagnosed with acne and treated with isotretinoin.

Methods: The study included 70 patients ( 59 females and 11 males, between ages 18 and 37 ) with moderate to severe acne vulgaris treated with isotretinoin. The medical records and laboratory findings of the participants were reviewed retrospectively between March 2017 and September 2018. All the patients whose necessary laboratory test results could be obtained from the collected data were included in the study.

Results: Serum total cholesterol, HDL, LDL, triglyceride, mean corpuscular hemoglobin levels, and platelet/lymphocyte ratio increased, whereas white blood cell count and mean platelet volume $(p=0.036)$ decreased after isotretinoin treatment.

Conclusions: The results of this study revealed that mean platelet volume decreased significantly 3 months after the initiation of isotretinoin treatment. Therefore, we suggest considering mean platelet volume as an inflammatory marker in patients with acne treated with isotretinoin. However, this research should be replicated under more randomized conditions in a prospective study to reach a definitive conclusion.
\end{abstract}

Keywords: acne vulgaris, cholesterol, complete blood count, inflammation, isotretinoin, mean platelet volume

Received: 7 February 2019| Returned for modification: 18 February 2019| Accepted: 17 April 2019

\section{Introduction}

Acne vulgaris is a common inflammatory skin disorder of the pilosebaceous units (1). Acne vulgaris affects $9.4 \%$ of the general population across the world. Acne is most common among teenagers. It is more frequent in men; however, its prevalence in women increases with age. Male patients are more likely to have severe acne compared to females (2). Etiological factors include increased sebum synthesis, hyperkeratosis in the follicular infundibulum, inflammation, and Cutibacterium acnes. Acne vulgaris lesions primarily occur on the face, and thus may negatively affect patients' quality of life. It has been suggested that acne can lead to psychological diseases such as anxiety, low self-worth, and depression among adolescents and adults (1).

Mild acne can be treated by topical agents including benzoyl peroxide and retinoids, and by antibiotics such as erythromycin or clindamycin. Systemic antibiotics such as doxycycline and minocycline are used in combination with topical medications in treating patients with moderate acne. First-line treatment options for severe acne include benzoyl peroxide, topical erythromycin/clarithromycin, topical retinoids, oral antibiotics, and oral isotretinoin (3). Oral contraceptives containing estrogen and spironolactone are regarded as second-line treatment choices in females with inflammatory acne $(3,4)$. Moreover, it has been suggested that pulsed dye laser and $1,450 \mathrm{~nm}$ diode laser could be therapeutic alternatives (5).

Isotretinoin is a systemic retinoid that is used in the treatment of both recalcitrant nodulocystic acne and moderate to severe acne unresponsive to oral antibiotics. Isotretinoin has both an anti-inflammatory effect and comedolytic activity. Moreover, isotretinoin reduces sebum production and Cutibacterium acnes concentration (1). Despite its anti-inflammatory effect by downregulating toll-like receptor 2 expression on monocytes and neutrophil migration, the exact mechanism of action of isotretinoin has not been clearly identified yet (6).

Isotretinoin is also used to treat various disorders such as rosacea, periorificial dermatitis, seborrheic dermatitis, folliculitis, granuloma annulare, sarcoidosis, and photoageing (7). The most common side effect of isotretinoin treatment is mucocutaneous toxicity. Mucocutaneous toxicity reveals itself as xerosis, cheilitis, dry eyes, epistaxis, and alopecia. Nevertheless, isotretinoin treatment has been associated with various complications, including blepharitis, papilledema, anorexia, nausea, abdominal pain, cirrhosis, headache, lethargy, depression, muscle pain, arthralgia, birth defects, elevated levels of triglycerides and cholesterol, and effects on liver function tests (8). Therefore laboratory tests, including lipid profile, hepatic panel, and a pregnancy test, have been recommended during isotretinoin use (9).

Systemic isotretinoin use has been implicated in the deterioration of the ovarian reserve, development or aggravation of inflammatory bowel diseases, depression, anxiety, and suicide attempts. It has been suggested that decreased hippocampal neurogenesis due to isotretinoin might result in isotretinoin-induced depression (10).

Isotretinoin use can lead to various abnormalities in hematological parameters, such as leukopenia, neutropenia, agranulocytosis, thrombocytopenia, and thrombocytosis $(11,12)$. The incidence and severity of alterations in hematological parameters are 
usually not significant (11). Routine monitoring of white blood cell count, hemoglobin level, and platelet count during isotretinoin therapy has been recommended for patients with a clinical suspicion of an abnormality (11).

On the other hand, serious medical conditions such as severe and prolonged thrombocytopenia have been reported due to isotretinoin treatment. It is recommended that a complete blood count be obtained before and throughout isotretinoin treatment for optimal safety $(12,13)$.

However, it is a matter of debate whether hematological parameters should be followed during isotretinoin treatment. Furthermore, no consensus exists on the frequency of monitoring complete blood count during the course of isotretinoin treatment (13).

This study examines changes in the blood chemistry panel, hematological parameters, and inflammation biomarkers of patients treated with isotretinoin. The results will hopefully contribute to the medical literature to define the required laboratory parameters and inflammation biomarkers during the follow-up to isotretinoin treatment.

\section{Methods}

This study was conducted retrospectively. We investigated 70 patients ( 59 females and 11 males, between ages 18 and 37) with moderate or severe acne vulgaris admitted to a dermatology outpatient clinic and treated with isotretinoin. All the patients were included in the study regardless of age, race, and sex. Patients that received at least 3 months of isotretinoin treatment for acne and whose necessary laboratory test results could be obtained from the data were included in the study. The diagnosis of acne was made based on patient history and physical examination. Medical records and laboratory findings of the participants between March 2017 and September 2018 were reviewed retrospectively. Therefore, neither personnel nor the participants were blinded. The orally administered dose of isotretinoin was $0.5 \mathrm{mg} / \mathrm{kg}$ per day. Laboratory test results of the patients were evaluated the day before the initiation of isotretinoin treatment, and also at the end of the zrd month. Fasting venous blood samples were obtained from all participants. Blood cell counts were analyzed using the Cell-Dyn Ruby system. Biochemical parameters were evaluated using a C-80oo analyzer (Abbott Diagnostics, USA). Hematological parameters and chemistry panels, including fasting plasma glucose, alanine aminotransferase (ALT), aspartate aminotransferase (AST), creatinine, and lipid profile, were evaluated. In addition, the neutrophil/lymphocyte, monocyte/lymphocyte, platelet/lymphocyte, mean platelet volume / platelet count, derived neutrophil / lymphocyte ratio [neutrophils / (white blood cells - neutrophils)], (neutrophil $\times$ monocyte) / lymphocyte, and (neutrophil $\times$ monocyte $\times$ platelet) / lymphocyte ratios were analyzed $(14,15)$. Severity of acne was determined using the global acne grading system (16). All the patients were previously treated with topical agents or systemic antibiotics. None of them were receiving co-medications. No specific protocol for food was applied during the study. The exclusion criteria were a hematological disorder, liver disease, kidney disease, chronic inflammatory disorder, malignancy, diabetes mellitus, hyperlipidemia, infectious disease, eating disorder, or special diet. Hospital staff were also excluded from the study. The study was not supported by any industry.

Statistical analysis was performed using the SPSS 20.0 statistical package program. Descriptive statistics were presented as number and percentage for classified data, and also as average and median for numerical values. The normal distribution of pretreatment and post-treatment numerical values was evaluated with the Kolmogorov-Smirnov test. The Wilcoxon signed-rank test (a non-parametric test) was used to compare the pre-treatment and post-treatment numerical values, which were not normally distributed. A $p$-value $<0.05$ was considered significant.

\section{Results}

This study included 70 patients with acne vulgaris: 59 (84.3\%) females and $11(15.7 \%)$ males. The mean age of the patients was $23.03 \pm 4.35$ years. The median (minimum-maximum) age of the patients was 22 (18-37).

Fasting plasma glucose levels, serum ALT, AST, and creatinine levels were similar before and after isotretinoin treatment. Nonetheless, serum total cholesterol, HDL cholesterol, LDL cholesterol, and triglyceride levels increased after isotretinoin treatment. $P$-values were $<0.001,0.025,<0.001$, and 0.007 , respectively. The values were not normally distributed (Wilcoxon signed-rank test). The mean fasting plasma glucose levels of the patients before and at the end of the 3rd month of isotretinoin therapy were 86.27 \pm 4.79 and $87.00 \pm 6.24 \mathrm{mg} / \mathrm{dl}$, respectively $(p=0.302$, reference ranges: $65-105 \mathrm{mg} / \mathrm{dl})$. The mean serum AST levels before and after therapy were $15.88 \pm 3.94$ and $16.12 \pm 3.33 \mathrm{U} / 1$, respectively ( $p$ $=0.353$, reference ranges: $5-40 \mathrm{U} / 1)$. The mean serum ALT levels before and after therapy were $13.84 \pm 6.52$ and $15.50 \pm 12.09 \mathrm{U} / \mathrm{l}$, respectively ( $p=0.122$, reference ranges: $0-55 \mathrm{U} / 1)$. The mean serum creatinine levels before and after therapy were $0.74 \pm 0.08$ and $0.73 \pm 0.08 \mathrm{mg} / \mathrm{dl}$, respectively ( $p=0.560$, reference ranges: $0.4-1.3 \mathrm{mg} / \mathrm{dl}$ ). The mean serum total cholesterol levels before and after therapy were $163.77 \pm 25.52$ and $175.00 \pm 30.24 \mathrm{mg} / \mathrm{dl}$, respectively ( $p<0.001$, reference ranges: $130-200 \mathrm{mg} / \mathrm{dl}$ ). The mean serum HDL cholesterol levels before and after therapy were 49.16 \pm 9.81 and $51.71 \pm 10.60 \mathrm{mg} / \mathrm{dl}$, respectively ( $p=0.025$, reference ranges: $40-70 \mathrm{mg} / \mathrm{dl}$ ). The mean serum LDL cholesterol levels before and after therapy were $84.15 \pm 19.96$ and $93.48 \pm 24.76 \mathrm{mg} /$ $\mathrm{dl}$, respectively ( $p<0.001$, reference ranges: $60-130 \mathrm{mg} / \mathrm{dl}$ ). The mean serum triglyceride levels were $76.38 \pm 29.72$ and $84.42 \pm 31.61$ $\mathrm{mg} / \mathrm{dl}$, respectively ( $p=0.007$, reference ranges: 50-150 mg/dl).

The results of the complete blood count revealed that red blood cell (RBC) count, serum hemoglobin, hematocrit, mean corpuscular volume (MCV) levels, mean corpuscular hemoglobin concentration (MCHC), red cell distribution width, and platelet count were similar before and after the treatment. White blood cell (WBC) count and mean platelet volume (MPV) decreased, but mean corpuscular hemoglobin $(\mathrm{MCH})$ levels increased after isotretinoin treatment. The neutrophil/lymphocyte, monocyte/lymphocyte, mean platelet volume / platelet count, neutrophil / (white blood cell - neutrophil), (neutrophil $\times$ monocyte) / lymphocyte, and (neutrophil $\times$ monocyte $\times$ platelet) $/$ lymphocyte ratios were similar before and after treatment. However, the platelet/lymphocyte ratio increased significantly after isotretinoin treatment.

The mean WBC counts before and after therapy were $7.39 \pm 1.50$ and $6.91 \pm 1.60 \times 10^{3} / \mu \mathrm{l}\left(p=0.001\right.$, reference ranges: $4.2-10.2 \times 10^{3} /$ $\mu \mathrm{l})$. The mean red blood cell (RBC) counts before and after therapy were $4.85 \pm 0.44$ and $4.83 \pm 0.50 \mathrm{M} / \mu \mathrm{l}(p=0.463$, reference ranges: 4-6.1 M/ $\mu \mathrm{l})$. The mean serum hemoglobin levels before and after therapy were $14.14 \pm 1.3$ and $14.19 \pm 1.17 \mathrm{~g} / \mathrm{dl}$ ( $p=0.725$, reference ranges: $11.7-17 \mathrm{~g} / \mathrm{dl})$. The mean hematocrit levels before and after therapy were $41.80 \pm 3.25 \%$ and $41.73 \pm 3.13 \%(p=0.921$, reference ranges: $35-51 \%)$. The MCV levels before and after therapy were 
$86.28 \pm 5.04$ and $86.70 \pm 4.51 \mathrm{fl}(p=0.071$, reference ranges: $80-100$ fl). The mean MCH levels before and after therapy were $29.20 \pm$ 2.32 and $29.65 \pm 2.43 \mathrm{pg}(p=0.005$, reference ranges: $27-34 \mathrm{pg})$. The MCHC before and after therapy was $33.82 \pm 1.22 \%$ and 33.99 $\pm 1.19 \%$ ( $p=0.216$, reference ranges: $31-38 \%)$. The mean red cell distribution width (RDW-SD) before and after therapy was $11.52 \pm$ $1.68 \%$ and $11.19 \pm 1.31 \%$ ( $p=0.147$, reference ranges: $10-17.6 \%$ ). The mean platelet count (PLT) before and after therapy was 270.06 \pm 52.11 and $270.23 \pm 56.15 \times 10^{3} / \mu \mathrm{l}(p=0.946$, reference ranges: $142-$ $\left.450 \times 10^{3} / \mu \mathrm{l}\right)$. The MPV before and after therapy was $8.18 \pm 1.36$ and $8.05 \pm 1.58 \mathrm{fl}$ ( $p=0.036$, reference ranges: 6.4-11 fl).

The percentages of lymphocytes, monocytes, neutrophils, eosinophils, and basophils, and the absolute counts of monocytes, neutrophils, eosinophils, and basophils were similar before and after isotretinoin treatment. Nevertheless, the absolute count of lymphocytes decreased significantly after isotretinoin treatment $(p<0.001$; Table 1$)$.

The neutrophil/lymphocyte ratio before and after therapy was $1.63 \pm 0.60$ and $1.74 \pm 0.6$, respectively $(p=0.163)$. The monocyte/ lymphocyte ratio before and after therapy was $0.20 \pm 0.04$ and 0.22 \pm 0.05 , respectively $(p=0.059)$. The platelet/lymphocyte (PLT/L) ratio before and after therapy was $109.01 \pm 32.54$ and $121.01 \pm$ 30.84, respectively $(p=0.001)$. The MPV/PLT count before and after therapy was $3.19 \pm 0.98$ and $3.15 \pm 1.12$ fl10 $-3 \mu \mathrm{l}-1$, respectively $(p=0.438)$. The derived neutrophil/lymphocyte ratio [neutrophils / (white blood cells - neutrophils)] before and after therapy was $1.26 \pm 0.45$ and $1.34 \pm 0.52$, respectively $(p=0.260)$. The (neutrophil $\times$ monocyte) / lymphocyte ratio before and after therapy was 0.88 \pm 0.44 and $0.90 \pm 0.44$, respectively $(p=0.699)$. The (neutrophil $\times$ monocyte $\times$ platelet) / lymphocyte ratio before and after therapy was $240.92 \pm 119.11$ and $246.51 \pm 140.44$, respectively $(p=0.937$; Table 2).

\section{Discussion}

Inflammation occurs in the development of all forms of acne le- sions, such as inflammatory papules, pustules, and nodules, and even during the early stages, such as microcomedones and comedones (17). Chemoattractants produced by bacteria in occluded sebaceous follicles lead to dermal neutrophil infiltration. Systemic retinoids inhibit follicular hyperkeratosis and sebum production, and they affect the bacterial population of sebaceous follicles. Norris et al. showed that isotretinoin had a potent antiinflammatory effect by inhibiting monocyte and neutrophil chemotaxis (18). Laboratory tests including liver enzymes and lipids should be checked through the course of isotretinoin treatment (19). Evaluation of changes in complete blood count has also been advised (20). However, there is no consensus on the frequency of monitoring complete blood count (21).

Studies investigating the effects of isotretinoin on liver enzymes, lipids, complete blood count, and inflammation markers have been reported. Brzezinski et al. followed up patients with peripheral blood morphology with smear, lipid profile, and liver function tests. Increased serum levels of total cholesterol and serum triglycerides were observed in $3.11 \%$ of patients, and increases in serum levels of liver enzymes after treatment were observed in $2.09 \%$ of patients. Brzezinski et al. reported that total cholesterol, serum triglycerides, and liver enzymes returned to normal limits within 2 to 3 weeks after cessation of isotretinoin (7). Waisman reported a 16-year-old male patient with agranulocytosis and elevated levels of AST and ALT as a result of isotretinoin therapy. The granulocyte count reached normal levels 4 weeks after the drug was discontinued (22). Hansen et al. reported insignificant leukopenia in $1.4 \%$ of patients and thrombocytopenia in $0.9 \%$ of patients receiving isotretinoin therapy for acne. However, significantly elevated triglyceride and cholesterol levels were observed in $19.3 \%$ and $22.8 \%$ of patients, respectively. Hansen et al. suggested that lipid panel and liver function tests should be checked 2 months after the initiation of isotretinoin therapy. Hansen et al. did not recommend further testing for patients with normal laboratory findings (23).

Lee et al. reviewed the laboratory changes in patients with acne

Table 1 | Complete blood count results of patients with acne before and after 3 months of isotretinoin treatment.

\begin{tabular}{|c|c|c|c|c|}
\hline Measure & Before isotretinoin treatment & After 3 months of isotretinoin treatment & Reference ranges & $p$ \\
\hline$\%$ of lymphocytes & $35.34 \pm 6.64$ & $34.01 \pm 6.48$ & $10-50 \%$ & 0.079 \\
\hline$\%$ of monocytes & $7.25 \pm 1.45$ & $7.48 \pm 1.55$ & $0-12 \%$ & 0.303 \\
\hline$\%$ of neutrophils & $54.27 \pm 8.17$ & $55.39 \pm 7.92$ & $37-80 \%$ & 0.242 \\
\hline$\%$ of eosinophils & $1.97 \pm 1.3$ & $1.92 \pm 1.23$ & $0-7 \%$ & 0.942 \\
\hline$\%$ of basophils & $1.14 \pm 0.37$ & $1.16 \pm 0.41$ & $0-2.5 \%$ & 0.772 \\
\hline Absolute count of lymphocytes & $2.58 \pm 0.52$ & $2.30 \pm 0.48$ & $0.6-3.4 \times 10^{3} / \mu \mathrm{l}$ & $<0.001$ \\
\hline Absolute count of monocytes & $0.53 \pm 0.12$ & $0.51 \pm 0.14$ & $0-0.9 \times 10^{3} / \mu \mathrm{l}$ & 0.127 \\
\hline Absolute count of neutrophils & $4.12 \pm 1.45$ & $3.89 \pm 1.29$ & $2-7 \times 10^{3} / \mu \mathrm{l}$ & 0.141 \\
\hline Absolute count of eosinophils & $0.14 \pm 0.09$ & $0.13 \pm 0.08$ & $0-0.7 \times 10^{3} / \mu \mathrm{l}$ & 0.091 \\
\hline Absolute count of basophils & $0.09 \pm 0.08$ & $0.07 \pm 0.02$ & $0-0.2 \times 10^{3} / \mu \mathrm{l}$ & 0.056 \\
\hline
\end{tabular}

The percentages of lymphocytes, monocytes, neutrophils, eosinophils, and basophils and absolute counts of monocytes, neutrophils, eosinophils, and basophils were similar before and after isotretinoin treatment in patients with acne. However, the absolute count of lymphocytes was significantly higher before treatment $(p<0.001)$.

Table 2 | Laboratory tests that changed significantly after isotretinoin treatment.

\begin{tabular}{|c|c|c|c|}
\hline & Before isotretinoin treatment & After isotretinoin treatment & $p$ \\
\hline Total cholesterol (mg/dl) & $163.77 \pm 25.52$ & $175.00 \pm 30.24$ & $<0.001$ \\
\hline HDL cholesterol (mg/dl) & $49.16 \pm 9.81$ & $51.71 \pm 10.60$ & 0.025 \\
\hline LDL cholesterol (mg/dl) & $84.15 \pm 19.96$ & $93.48 \pm 24.76$ & $<0.001$ \\
\hline Triglyceride (mg/dl) & $76.38 \pm 29.72$ & $84.42 \pm 31.61$ & 0.007 \\
\hline WBC $\left(\times 10^{3} / \mu \mathrm{l}\right)$ & $7.39 \pm 1.50$ & $6.91 \pm 1.60$ & 0.001 \\
\hline $\mathrm{MCH}(\mathrm{pg})$ & $29.20 \pm 2.32$ & $29.65 \pm 2.43$ & 0.005 \\
\hline MPV (fl) & $8.18 \pm 1.36$ & $8.05 \pm 1.58$ & 0.036 \\
\hline Absolute count of lymphocytes $\left(\times 10^{3} / \mu \mathrm{l}\right)$ & $2.58 \pm 0.52$ & $2.30 \pm 0.48$ & $<0.001$ \\
\hline $\mathrm{PLT} / \mathrm{L}$ & $109.01 \pm 32.54$ & $121.01 \pm 30.84$ & 0.001 \\
\hline
\end{tabular}

$\overline{\mathrm{WBC}}=$ white blood cell count, $\mathrm{MCH}=$ mean corpuscular hemoglobin, $\mathrm{MPV}=$ mean platelet volume, $\mathrm{PLT} / \mathrm{L}=\mathrm{platelet} / \mathrm{lymphocyte}$ ratio.

Serum levels of total cholesterol, HDL cholesterol, LDL cholesterol, triglyceride, MCH, and PLT/L increased. However, WBC, MPV, and absolute count of lymphocytes decreased by the end of the 3rd month of systemic isotretinoin treatment. 
vulgaris during isotretinoin therapy. Isotretinoin was associated with a statistically significant decrease in WBC count. However, hemoglobin, hematocrit, and PLT counts could not be analyzed as a result of lack of data (9). Michaëlsson et al. reported that isotretinoin therapy at a dose of $0.5 \mathrm{mg} / \mathrm{kg} /$ day for 3 months led to a significant decrease in WBC count, especially in the number of neutrophils. The observed changes were dose-dependent and reversible (24). Karadag et al. reported an increase in PLT count after 3 months of isotretinoin therapy. Posttreatment hemoglobin, hematocrit, and WBC counts were observed to be similar with baseline results (25). Ataseven et al. associated isotretinoin therapy with significantly decreased PLT counts and MPV (26). Gencoglan et al. also evaluated hematological parameters during isotretinoin treatment in patients with acne vulgaris. WBC count and neutrophils decreased in the 1st month, increased in the 2nd month, and decreased again at the end of the treatment. Platelet count increased in the 1st month of the treatment and then it decreased. MCV increased at the end of the treatment (27).

Seckin et al. investigated the effect of isotretinoin on inflammatory markers and hematological parameters. Hemoglobin levels and platelet counts increased and red blood cell distribution width decreased significantly 3 months after isotretinoin therapy. Moreover, neutrophil lymphocyte rate decreased, and platelet lymphocyte rate was increased by isotretinoin. However, these changes were not statistically significant (28). Ozuguz et al. suggested that isotretinoin might increase the monocyte count and decrease hemoglobin levels, red blood cell count, and MCV. However, Ozuguz et al. did not observe a significant difference in WBC, neutrophil count, lymphocyte count, and neutrophil/lymphocyte ratio in 3 months of isotretinoin therapy (29). Similarly, Ataseven et al. reported no significant differences in neutrophil count, lymphocyte count, and neutrophil/lymphocyte ratio in patients with acne vulgaris receiving isotretinoin (30).

In this study, fasting plasma glucose, AST, ALT, and creatinine levels were similar 3 months after isotretinoin therapy compared to basal levels. However, serum levels of total cholesterol, HDL cholesterol, LDL cholesterol, and triglyceride showed a significant increase with isotretinoin therapy. None of the patients had nutrition recommendations or dietary restrictions. In addition, eating habits could not be evaluated during this study. Therefore, high serum lipid levels after isotretinoin treatment might have been affected by dietary habits. The changes we observed in the complete blood count values were a statistically significant increase in $\mathrm{MCH}$ and a significant decrease in WBC counts, absolute lymphocyte counts, and MPV. Complete blood count-derived inflammation biomarkers, including neutrophil/lymphocyte, monocyte/lymphocyte, MPV/PLT, derived neutrophil/lymphocyte ratio, (neutrophil $\times$ monocyte) / lymphocyte, and (neutrophil $\times$ monocyte $\times$ platelet) / lymphocyte ratio, did not show significant difference with isotretinoin therapy. However, we observed a statistically significant increase in PLT/L.

MPV has been regarded as an inflammatory marker in various chronic diseases (31). In this study, MPV decreased significantly 3 months after the initiation of isotretinoin. Therefore, we suggest that MPV may be considered an inflammatory marker in patients with acne. However, complete blood count-derived inflammation biomarkers were similar with isotretinoin therapy except for PLT/L. The PLT/L ratio might be increased as a result of decreased absolute lymphocyte counts.

The main limitations of this study were not evaluating patients' eating habits and conducting the study retrospectively. This research should be replicated under more randomized conditions in a prospective study.

\section{Conclusions}

In conclusion, our results revealed that isotretinoin therapy led to a decrease in WBC count, absolute lymphocyte count, and MPV in patients with acne. Therefore, we suggest that complete blood count should be checked in every patient treated with isotretinoin. Moreover, we suggest considering MPV as an inflammatory marker in patients with acne treated with isotretinoin, whereas an increasing or decreasing MPV value might help the clinician evaluate the effectiveness of the treatment. In addition, PLT/L was the only complete blood count-derived inflammation biomarker that was significantly affected by isotretinoin, which encouraged us to use PLT/L together with MPV to evaluate the course of action of isotretinoin for further studies.

\section{References}

1. Zaenglein AL. Acne vulgaris. N Engl J Med. 2018;379:1343-52.

2. Tan JK, Bhate K. A global perspective on the epidemiology of acne. Br J Dermatol. 2015;172:3-12.

3. Hauk L. Acne vulgaris: treatment guidelines from the AAD. Am Fam Physician. 2017;95:740-1.

4. Kraft J, Freiman A. Management of acne. CMAJ. 2011;183:430-5.

5. Barbieri JS, Spaccarelli N, Margolis DJ, James WD. Approaches to limit systemic antibiotic use in acne: systemic alternatives, emerging topical therapies, dietary modification, and laser and light-based treatments. J Am Acad Dermatol. 2019;80:538-49.

6. Kelhälä HL, Fyhrquist N, Palatsi R, Lehtimäki S, Väyrynen JP, Kubin ME, et al. Isotretinoin treatment reduces acne lesions but not directly lesional acne inflammation. Exp Dermatol. 2016;25:477-8.

7. Brzezinski P, Borowska K, Chiriac A, Smigielski J. Adverse effects of isotretinoin: a large, retrospective review. Dermatol Ther. 2017;30:12483.

8. Ellis CN, Krach KJ. Uses and complications of isotretinoin therapy. J Am Acad Dermatol. 2001;45:150-7.

9. Lee YH, Scharnitz TP, Muscat J, Chen A, Gupta-Elera G, Kirby JS. Laboratory monitoring during isotretinoin therapy for acne: a systematic review and metaanalysis. JAMA Dermatol. 2016;152:35-44.

10. Melnik BC. Apoptosis may explain the pharmacological mode of action and adverse effects of isotretinoin, including teratogenicity. Acta Derm Venereol. 2017;97:173-81.

11. Zane LT, Leyden WA, Marqueling AL, Manos MM. A population-based analysis of laboratory abnormalities during isotretinoin therapy for acne vulgaris. Arch Dermatol. 2006;142:1016-22.

12. Moeller KE, Touma SC. Prolonged thrombocytopenia associated with isotretinoin. Ann Pharmacother. 2003;37:1622-4.

13. Jones MC. Update on isotretinoin and the iPLEDGE system. US Pharm. 2007;32: $38-46$.

14. Djordjevic D, Rondovic G, Surbatovic M, Stanojevic I, Udovicic I, Andjelic T, et al. Neutrophil-to-lymphocyte ratio, monocyte-to-lymphocyte ratio, platelet-tolymphocyte ratio, and mean platelet volume-to-platelet count ratio as biomarkers in critically ill and injured patients: which ratio to choose to predict outcome and nature of bacteremia? Mediators Inflamm. 2018;2018:3758068.

15. Pinna A, Porcu T, D’Amico-Ricci G, Dore S, Boscia F, Paliogiannis P, et al. Complete blood cell count-derived inflammation biomarkers in men with age-related macular degeneration. Ocul Immunol Inflamm. 2018; [Epub ahead of print].

16. Adityan B, Kumari R, Thappa DM. Scoring systems in acne vulgaris. Indian J Dermatol Venereol Leprol. 2009;75:323-6.

17. Tanghetti EA. The role of inflammation in the pathology of acne. J Clin Aesthet Dermatol. 2013;6:27-35.

18. Norris DA, Osborn R, Robinson W, Tonnesen MG. Isotretinoin produces significant inhibition of monocyte and neutrophil chemotaxis in vivo in patients with cystic acne. J Invest Dermatol. 1987;89:38-43 
19. Layton A. The use of isotretinoin in acne. Dermatoendocrinol. 2009;1:162-9.

20. Strauss JS, Krowchuk DP, Leyden JJ, Lucky AW, Shalita AR, Siegfried EC, et al. Guidelines of care for acne vulgaris management. J Am Acad Dermatol. 2007; 56:651-63.

21. Goldsmith LA, Bolognia JL, Callen JP, Chen SC, Feldman SR, Lim HW, et al. American Academy of Dermatology Consensus Conference on the safe and optimal use of isotretinoin: summary and recommendations. J Am Acad Dermatol. 2004;50:900-6.

22. Waisman M. Agranulocytosis from isotretinoin. J Am Acad Dermatol. 1988;18: 395-6.

23. Hansen TJ, Lucking S, Miller JJ, Kirby JS, Thiboutot DM, Zaenglein AL. Standardized laboratory monitoring with use of isotretinoin in acne. J Am Acad Dermatol. 2016;75:323-8.

24. Michaëlsson G, Vahlquist A, Mobacken H, Hersle K, Landegren J, Rönnerfält L, et al. Changes in laboratory variables induced by isotretinoin treatment of acne. Acta Derm Venereol. 1986;66:144-8.

25. Karadag AS, Ertugrul DT, Takci Z. Isotretinoin modestly increases platelet coun in acne patients. J Dermatolog Treat. 2013;24:139-40.
26. Ataseven A, Ugur Bilgin A. Effects of isotretinoin on the platelet counts and the mean platelet volume in patients with acne vulgaris. ScientificWorldJournal. 2014;2014:156464.

27. Gencoglan G, Inanir I, Miskioglu M, Gunduz K. Evaluation of sequential effect of isotretinoin on the haematological parameters in patients with acne vulgaris. Cutan Ocul Toxicol. 2018;37:139-42.

28. Seckin HY, Bas Y, Takcı Z, Kalkan G. Effects of isotretinoin on the inflammatory markers and the platelet counts in patients with acne vulgaris. Cutan Ocul Toxicol. 2016;35:89-91.

29. Ozuguz P, Dogruk Kacar S, Polat S, Ulu S, Karaca S. Evaluation of the efficacy of isotretinoin treatment on neutrophil/lymphocyte ratio and the other hemogram parameters. Mustafa Kemal Univ Tip Derg. 2013;13:9-14.

30. Ataseven A, Saylam Kurtipek G, Ozturk P. Neutrophil lymphocyte ratio in patients receiving isotretinoin for acne vulgaris. Med Science. 2014;3:1026-31.

31. Wang X, Meng H, Xu L, Chen Z, Shi D, Lv D. Mean platelet volume as an inflammatory marker in patients with severe periodontitis. Platelets. 2015;26:67-71. 